\title{
Evaluation of the three methods of bacterial decontamination on implants with three different surfaces
}

\author{
Paweł Kubasiewicz-Ross 1,A,C,D, Małgorzata Fleischer ${ }^{2, B, C}$, Artur Pitułaj, ${ }^{1, A-C}$, Jakub Hadzik ${ }^{1, A, D, F}$, \\ Izabela Nawrot-Hadzik ${ }^{3, E}$, Olga Bortkiewicz ${ }^{2, B, C}$, Marzena Dominiak ${ }^{1, F}$, Kamil Jurczyszyn ${ }^{1, A-C, E}$ \\ ${ }^{1}$ Department of Oral Surgery, Wroclaw Medical University, Poland \\ 2 Department of Microbiology, Wroclaw Medical University, Poland \\ ${ }^{3}$ Department of Biology and Pharmaceutical Botany, Wroclaw Medical University, Poland \\ A - research concept and design; $B$ - collection and/or assembly of data; $C$ - data analysis and interpretation; \\ $D$ - writing the article; $E$ - critical revision of the article; $F$ - final approval of the article
}

Address for correspondence

Paweł Kubasiewicz-Ross

E-mail: pawelkubasiewicz@wp.pl

Funding sources

None declared

Conflict of interest

None declared

Received on March 13, 2019

Reviewed on March 28, 2019

Accepted on September 25, 2019

Published online on February 25, 2020

Cite as

Kubasiewicz-Ross P, Fleischer M, Pitułaj A, et al. Evaluation of the three methods of bacterial decontamination on implants with three different surfaces. Adv Clin Exp Med. 2020:29(2):177-182. doi:10.17219/acem/112606

DOI

10.17219/acem/112606

Copyright

Copyright by Author(s)

This is an article distributed under the terms of the

Creative Commons Attribution 3.0 Unported (CC BY 3.0)

(https://creativecommons.org/licenses/by/3.0/)

\begin{abstract}
Background. The main goal of the treatment of the peri-implantitis is to decontaminate the surface of the implant, thereby enabling further treatment involving, e.g., guided bone regeneration. Since new implants of the rougher surface were introduced to the common dental practice, decontamination is even more difficult.
\end{abstract}

Objectives. The aim of the study was to evaluate 3 different methods of decontaminating implants with 3 different surfaces.

Material and methods. A total of 30 dental implants with 3 different surface types (machined, sandblasted, and acid-etched (SLA) and hydroxyapatite (HA)-coated) were used in the study. Each group of implants was coated with Escherichia coli biofilm and cultivated. Afterwards, the implants were transferred to the jaw model and treated with a different method: sonic scaler mechanical debridement with a Woodpecker PT5 sonic scaler ( ${ }^{\text {st }}$ group), and mechanical debridement with sonic scaler and with the combination with chemical agent Perisolv ${ }^{\circledR}$ (2nd group), and with Er:YAG laser treatment ( $3^{\text {rd }}$ group). Each implant was treated with the specific method and sent for further microbiological evaluation.

Results. The highest level of decontamination was achieved for machined-surface implants and the lowest for HA-coated implants. The method with the highest biofilm reduction was the scaler and Perisolv ${ }^{\circledR}$ group. The highest level of decontamination of HA-coated implants were achieved for Er:YAG laser irradiation method.

Conclusions. In the following paper, the superiority of combined chemical-mechanical method of decontaminating the surface of the implant on SLA and machined-surface implants was proved. On the contrary, Er:YAG laser irradiation was reported as the best option for decontamination of the HA-coated implants. In our opinion, it is a significant finding, revealing that the method of peri-implantitis management should be considered in accordance to the type of the surface of the implant (customized to the surface of the implant).

Key words: implant surface, implant surface treatment, bacterial coating 


\section{Introduction}

With the increasing number of patients treated with dental implants, a corresponding number of post-treatment complications can be expected. The most common complication in dental implant therapy is peri-implantitis. ${ }^{1}$ It is defined as an inflammatory reaction that affects the hard and soft tissue, which results in the loss of supporting bone and gingival pocket formation surrounding the functioning osseointegrated implant. ${ }^{2}$ This pathological condition is caused by a polymicrobial aggressive biofilm that colonizes the implant and abutment surface at the peri-implant crevice level. It is reported that its prevalence can rise up to $56 \%{ }^{1-3}$ Anaerobic Gram-negative organisms are most commonly found in peri-implantitis-affected sites and include in among others: Aggregatibacter actinomycetemcomitans, Porphyromonas gingivalis, Peptostreptococcus micros, Campylobacter rectus, Fusobacterium spp., and Prevotella intermedia, although there are also studies reporting the role of enteric rods (mostly Escherichia coli and Enterobacter cloace) in this pathology, especially at its early stage. ${ }^{4,5}$

Because of its complexity, peri-implantitis is still challenging to treat. Treatment involves decontamination and guided bone and tissue regeneration techniques. The decontamination process is especially difficult because the method applied can destroy the fragile surface of the implant. For this purpose a number of mechanical interventions (e.g., abrasive air powder, Teflon and plastic curettes, ultrasonic devices) and chemical agents (e.g., chlorhexidine, hydrogen peroxide) solely or in combination have been described as methods for implant surface decontamination. Although all mentioned procedures result in compromise, a successful gold standard method has not been yet established. An acceptable cleaning technique must be able to debride and detoxify the surface without traumatizing it. Decontamination with a laser, photodynamic therapy (PDT) and the application of chlorhexidine ( $\mathrm{CHX})$ does not seem to alter the surfaces of the dental implants. However, PDT can make an adhesive layer on the surface of the treated implants, which can facilitate new plaque formation. ${ }^{6,7}$ Recent studies have reported that lasers can also be used in peri-implantitis management. Previously, high-power $\mathrm{CO}_{2}$, diode and erbium lasers were used frequently, due to their hemostatic properties, selective calculus ablation and bactericidal effects. However, high-power lasers can cause an undesired increase of temperature and have been recently replaced by Er:YAG laser. Another disadvantage of lasers is the high cost of equipment. ${ }^{8-10}$

Dental implant surface decontamination has become even more complicated since the introduction of dental implants with improved osteoconductive properties. Machined-surface implants, which have been used for decades, have been replaced by implants characterized by a rougher surface. There are 2 main paths that can be followed in order to improve the osteoconductivity of the titanium implants. These approaches can be classified into the following 2 techniques: metallic implants are coated with the bioactive compounds that accelerate bone formation or a rough surface is formed directly on the metallic implants. ${ }^{11}$ Both techniques increase the roughness of the surface of the implant, making osteointegration more favorable. However, as a result, it facilitates biofilm formation on dental implant surfaces. ${ }^{1,2,3,6}$ To the best of our knowledge, there are very few studies that evaluate various decontamination methods on different surfaces of the implants.

\section{Material and methods}

The study was conducted on a total number of 30 dental implants. Implants were divided into 3 equal groups with 10 implants in each group. All the implants had the same length and diameter of L12Ø4 $\mathrm{mm}$. The $1^{\text {st }}$ group was machined-surface (M) implants (SGS Dental Implant System Holding - Zn, St. Gallen, Switzerland). In the $2^{\text {nd }}$ group, Denium Superline II (Dentium, Seoul, South Korea) sandblasted and acid-etched dental implants (SLA) were used. The $2^{\text {rd }}$ group (HA) included the hydroxyapatite (HA)-coated dental implants (SGS Dental Implant System Holding - Zn).

\section{Bacterial cultivation}

Peri-implantitis is caused by Gram-negative and anaerobic bacteria and E. coli were used as a model for Gramnegative bacteria. The reasons we did so is that there are many studies involving bacterial adhesion and decontamination carried out on dental implants with E. coli as bacteria of choice, as well as because it is a readily available and easily cultivated aerobic microorganism.

\section{Material}

The McConkey's medium (BioMaxima SA, Lublin, Poland); Sugar broth (BioMaxima SA); Saponin (SigmaAldrich, St. Louis, USA); reference strain: E. coli ATCC 25922.

\section{Conduct of the experiment}

\section{Preparation of the inoculums}

The E. coli ATCC 25922 strain from McConkey's medium was seeded into sugar broth and incubated at $37^{\circ} \mathrm{C}$ for $24 \mathrm{~h}$. From the obtained culture in sugar broth, an inoculum with a density of 0.5 on the McFarland Scale (MFa) was prepared.

\section{Implants coating}

The inoculum prepared in this way, in the amount of $500 \mu \mathrm{L}$, was inoculated with $50 \mathrm{~mL}$ of sugar broth. Then, the implant was aseptically inserted and the whole was incubated at $37^{\circ} \mathrm{C}$ for $24 \mathrm{~h}$. 


\section{Preparation of implants for further tests}

After this time, the implants were removed from the culture and rinsed 3 times, in each case, in $10 \mathrm{~mL}$ of sterile saline to remove the plankton forms of the culture, leaving only the biofilm formed by E. coli on the surface. Such prepared implants were transferred to the Department of Oral Surgery for further tests.

\section{Model of the jaw}

Before the decontamination process, each implant was placed in peri-implantitis jaw model. The model was made from acrylonitrile butadiene styrene (ABS) which is a common thermoplastic polymer. According to the cumulative interceptive supportive therapy (CIST) protocol, ${ }^{12}$ mechanical debridement and surgical operation classification is needed when the bone loss depth is greater than $5 \mathrm{~mm}$. Following this standard, 6-millimiter bone loss depth was defined in our model. The artificial bone defect was created by removing of the material with the calibrated trephine drill around the implant side.

\section{Decontamination protocols}

Every group of implants was decontaminated with 3 different methods. Before the decontamination process, each implant was placed in peri-implantitis model. Different protocols of implant surface decontamination were used in the study:

- Sonic scaler mechanical debridement with a Woodpecker PT5 sonic scaler (Woodpecker, Guilin, China) (s). Each implant was treated with a sonic device for $2 \mathrm{~min}$ alone (Fig. 1).

- Mechanical debridement with sonic scaler and with the combination with chemical agent Perisolv ${ }^{\circledR}$ (Regedent AG, Zurich, Switzerland). Each implant was pre-treated with Perisolv ${ }^{\circledR}$ application for $30 \mathrm{~s}$, then sonic scaler was applied for $2 \mathrm{~min}(\mathrm{~s}+\mathrm{p}$ ) (Fig. 2).

- Er:YAG laser treatment. Implants were decontaminated with Er-YAG (LiteTouch ${ }^{\mathrm{T}}$, Yokneam, Israel) laser irradiation with a $1.3 \times 17 \mathrm{~mm}$ tip, working up and down continuously for $2 \mathrm{~min}$, and the laser beam parameters were set for $40 \mathrm{~mJ}, 0.80 \mathrm{~W}, 20 \mathrm{~Hz}$ (Er:YAG) (Fig. 3).

Each implant was treated with the specific method and sent for further microbiological evaluation. The procedure for each implant was repeated 3 times and the results were averaged.

\section{Quantitative evaluation of microorganisms present in the biofilm on the implants surface}

Biofilm from the surface of the implants was removed with the use of an aqueous saponin solution. The implants (each separately) were placed in $1 \mathrm{~mL}$ of $0.5 \%$ saponin solution and shaken for $1 \mathrm{~min}(2,500 \mathrm{rpm}$; Heidolph

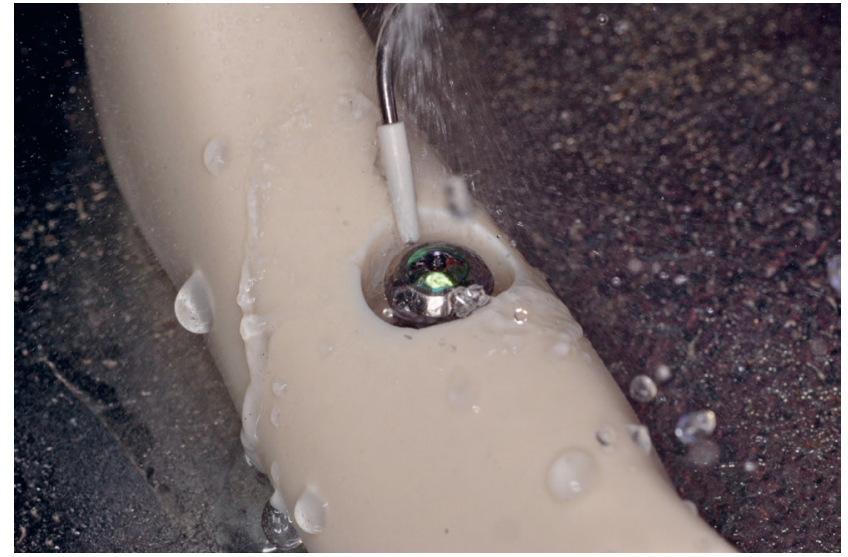

Fig. 1. Sonic scaler mechanical debridement

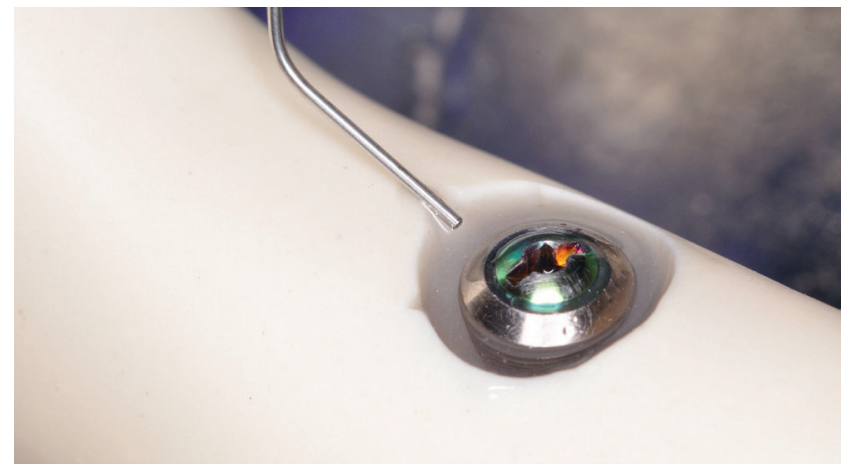

Fig. 2. Application of the Perisolv ${ }^{\circledR}$



Fig. 3. Er:YAG laser irradiation

Reax Control; Heidolph Instruments GmbH \& CO. KG, Schwabach, Germany). The obtained suspension of strains (saponin solution and bacteria suspended in it, detached from the surface of the implant) was immediately cultured on McConkey's medium. In the inoculation of bacteria, undiluted suspension was used, and suspension with dilutions from 1:10 to 1:1,000 inoculating volume: $10 \mathrm{~L}, 20 \mathrm{~L}$, $50 \mathrm{~L}$, and $100 \mathrm{~L}$. In order to obtain maximum separation of the biofilm, the procedure of its removal was repeated 3 times. Inoculated plates with McConkey's medium were incubated at $37^{\circ} \mathrm{C}$ for $22-24 \mathrm{~h}$. 


\section{Reading the results}

After incubation, the colonies grown on the plates were counted and the results obtained were given as the number of colony-forming units (CFU) per $1 \mathrm{~mL}$. The percentage of biofilm reduction $\mathrm{R}$ [\%] after the tested factor of biofilm removal acted on was calculated according to the formula:

$$
\mathrm{R}=\left[\left(\mathrm{S}_{\mathrm{C}}-\mathrm{S}\right) / \mathrm{S}_{\mathrm{C}}\right] \cdot 100 \%,
$$

where $\mathrm{S}_{\mathrm{C}}(\mathrm{CFU} / \mathrm{mL})$ - the total number of $E$. coli cells detached from the implant coating biofilm without the test factor acting (number of CFU/mL on the control implant);

$\mathrm{S}(\mathrm{CFU} / \mathrm{mL})$ - the total number of $E$. coli cells detached from the implant coating biofilm, which remain after the test factor acted.

In addition, to compare and reduce the measurement error, the degree of biofilm reduction was calculated after the rejection of extreme values:

$$
R^{\prime}=\left[\left(S_{C}^{\prime}-S^{\prime}\right) / S_{C}^{\prime}\right] \cdot 100 \%,
$$

where $S_{C}{ }_{C}(C F U / m L)$ - the total number of E. coli cells detached from the implant coating biofilm without the test factor acting (number of CFU/mL on the control implant), with no maximum or minimum value;

$\mathrm{S}^{\prime}(\mathrm{CFU} / \mathrm{mL})$ - the total number of $E$. coli cells detached from the implant coating biofilm, which remain after the test factor acted, with no maximum or minimum value.

\section{Statistical analysis}

Two-way analysis of variance (ANOVA) and Tukey's post hoc test were performed. All data is given as means \pm standard deviation (SD). A p-value $<0.05$ was considered statistically significant. The results were analyzed with STATISTICA v. 13 (StatSoft Poland, Kraków, Poland).

\section{Results}

The highest level of biofilm reduction ( $\left.R^{\prime}\right)$ for both mechanical and combined mechanical and chemical methods of decontamination was achieved for machined-surface implants $(98.66 \% \pm 1.19 \%$ (for s) and $98.61 \% \pm 1.39 \%$ (for $\mathrm{s}+\mathrm{p})$ ) and for SLA implants $(96.86 \% \pm 2.81 \%$ (for s) and $95.23 \% \pm 4.68 \%$ (for $\mathrm{s}+\mathrm{p}$ )) (Fig. 4-6). Taking under consideration all of the methods, there were no statistically important differences between decontamination of M and SLA group of implants, although there were statistically important differences between M and HA as well as SLA and HA groups. Surprisingly, as it can be seen, additional application of the chemical agent did not improve decontamination of machined-surface and SLA implants, although it significantly improved the decontamination of HA-coated implants $(78.82 \% \pm 13.69 \%$ (for s) while $85.26 \% \pm 19.65 \%$ (for $\mathrm{s}+\mathrm{p})$ ). Also, what is worth reporting are the unstable results in that group of implants (Fig. 6).

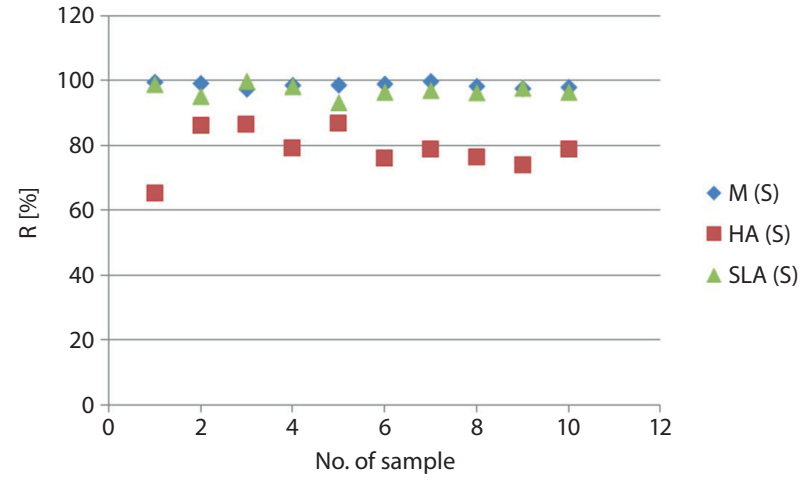

Fig. 4. The results of scaler application on the decontamination of the implant

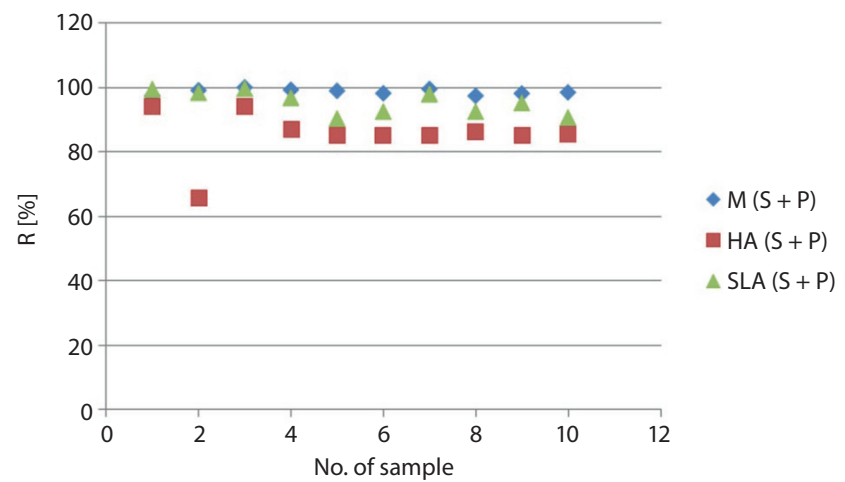

Fig. 5. The results of combined application of scaler and Perisolv application on the decontamination of the implant



Fig. 6. The results of Er:YAG application on the decontamination of the implant

However, HA-coated implants demonstrate a level of biofilm reduction after Er:YAG irradiation that is better than machined-surface implants and lower than SLA implants $(89.99 \% \pm 21.52 \%$ for HA, $87.40 \% \pm 1.49 \%$ for $\mathrm{M}$ and $95.98 \% \pm 5.45 \%$ for SLA). Taking under consideration the method of decontamination, the best results were achieved for combined mechanical and chemical $(\mathrm{s}+\mathrm{p})$ and this is the treatment of choice for SLA and M surfaced implants. The mechanical debridement reveals comparable to laser irradiation results. Laser application is also the method of treatment for HA implants decontamination.

The intra- and extragroup statistical analysis is shown in Table 1 . The statistically important differences are in bold. 
Table 1. Differences in the percentage of biofilm reduction between applied methods in relation to the implant surface

\begin{tabular}{|c|c|c|c|c|c|c|c|c|c|c|}
\hline $\begin{array}{l}\text { Biofilm } \\
\text { reduction }\end{array}$ & & M; Scal & $\begin{array}{c}M ; \\
\text { Scal.+Perisolv }\end{array}$ & $\begin{array}{l}\text { M; Laser } \\
\text { Er:YAG }\end{array}$ & $\begin{array}{l}\mathrm{HA} ; \\
\text { Scaler }\end{array}$ & $\begin{array}{c}\text { HA; } \\
\text { Scal.+Perisolv }\end{array}$ & $\begin{array}{l}\text { HA; Laser } \\
\text { Er:YAG }\end{array}$ & $\begin{array}{l}\text { SLA; } \\
\text { Scal. }\end{array}$ & $\begin{array}{c}\text { SLA; } \\
\text { Scal.+Perisolv }\end{array}$ & $\begin{array}{l}\text { SLA; Laser } \\
\text { Er:YAG }\end{array}$ \\
\hline $98.7 \%$ & $\begin{array}{l}\text { M; } \\
\text { Scal. }\end{array}$ & & 1.000 & $<0.05$ & $<0.05$ & $<0.05$ & $<0.05$ & 0.997 & 0.876 & 0.968 \\
\hline $98.6 \%$ & $\begin{array}{l}\text { M; } \\
\text { Scal.+Perisolv }\end{array}$ & 1.000 & & $<0.05$ & $<0.05$ & $<0.05$ & $<0.05$ & 0.998 & 0.885 & 0.971 \\
\hline $87.4 \%$ & $\begin{array}{l}\text { M; } \\
\text { Laser Er:YAG }\end{array}$ & $<0.05$ & $<0.05$ & & $<0.05$ & 0.992 & 0.974 & $<0.05$ & $<0.05$ & $<0.05$ \\
\hline $78.8 \%$ & $\begin{array}{l}\text { HA; } \\
\text { Scal. }\end{array}$ & $<0.05$ & $<0.05$ & $<0.05$ & & 0.159 & $<0.05$ & $<0.05$ & $<0.05$ & $<0.05$ \\
\hline $85.3 \%$ & $\begin{array}{l}\text { HA; } \\
\text { Scal.+Perisolv }\end{array}$ & $<0.05$ & $<0.05$ & 0.992 & 0.159 & & 0.553 & $<0.05$ & $<0.05$ & $<0.05$ \\
\hline $90.0 \%$ & $\begin{array}{l}\text { HA; } \\
\text { Laser Er:YAG }\end{array}$ & $<0.05$ & $<0.05$ & 0.974 & $<0.05$ & 0.553 & & 0.106 & 0.413 & 0.238 \\
\hline $96.9 \%$ & $\begin{array}{l}\text { SLA; } \\
\text { Scal. }\end{array}$ & 0.997 & 0.998 & $<0.05$ & $<0.05$ & $<0.05$ & 0.106 & & 0.998 & 0.999 \\
\hline $95.2 \%$ & $\begin{array}{l}\text { SLA; } \\
\text { Scal.+Perisolv }\end{array}$ & 0.876 & 0.885 & $<0.05$ & $<0.05$ & $<0.05$ & 0.413 & 0.998 & & 0.999 \\
\hline $96.0 \%$ & $\begin{array}{l}\text { SLA; } \\
\text { Laser Er:YAG }\end{array}$ & 0.968 & 0.971 & $<0.05$ & $<0.05$ & $<0.05$ & 0.238 & 0.999 & 0.999 & \\
\hline
\end{tabular}

M - machined-surface implants; HA - hydroxyapatite-coated implants; SLA - sandblasted and acid-etched implants. Differences statistically important are in bold at $\mathrm{p} \leq 0.05$.

\section{Discussion}

The methods used to decontaminate the surface of dental implants can be divided into 3 main groups. The $1^{\text {st }}$ group is comprised of the mechanical methods, the $2^{\text {nd }}$ group is comprised of the methods based on application of chemical agent on the implant surface and within the periodontal sulcus, and the $3^{\text {rd }}$ group includes physical methods (e.g., PDT or laser application). The mechanical debridement is also often an introduction to further therapy and is even considered as priority method. ${ }^{13-15}$

Mechanical debridement was also the first method used to manage peri-implantitis. The methods imported directly from the treatment of periodontitis were rather disappointing and resulted in damage to the fragile implant surface. That was the reason why alternative decontamination methods in dental implantology were sought. Mengel et al. were one of the first to evaluate several methods of mechanical debridement and to prove their safe applicability to fragile implant surface in an in vitro study. ${ }^{7}$ Blasi et al. provided the evaluation of different mechanical methods including ultrasonic scalers with plastic tips, titanium curettes, and airflow with glycine powder and with rubber cup and polishing paste. The study was conducted on patients suffering from severe (including CIST criteria) peri-implantitis and/ or mucosistis. They proved no statistically important difference between all 4 mechanical methods of implant surface decontamination. ${ }^{16}$

Denisson et al. were one of the first to design a study to evaluate the mechanical and chemical decontamination method of the dental implants with different surfaces. ${ }^{17}$
The dental implants were coated with radioactive endotoxin (125I-LPS). In an in vitro study, an air-powder abrasive with sodium bicarbonate as well as citric acid solution (CA), or $0.12 \% \mathrm{CHX}$ was used as a decontamination method on 2 different dental implant surfaces (titanium plasma-sprayed (TPS), HA-coated and machined-surface). As a result, they found the superiority of chemical methods. It was also found that machined-surface implants were decontaminated more effectively than the other surfaces by all treatments. The only exception for this statement was citric acid treatment, which was equally effective on either machined or hydroxyapatite surfaces.

The superiority of the chemical method in comparison to other methods of dental surface decontamination was reconfirmed in the study of Marotti et al. In an in vitro study, SLA implants were contaminated with the saliva collected from patients experiencing peri-implantitis. Several decontamination methods were applied, including the application of $0.12 \% \mathrm{CHX}, \mathrm{GaAlAs}$ laser irradiation $(660 \mathrm{~nm}$, $30 \mathrm{~mW}$ ) for $3 \mathrm{~min}$ or $5 \mathrm{~min}(7.2 \mathrm{~J}$ and $12 \mathrm{~J})$ without and with methylene blue dyes in PDT. They achieved greater decontamination in CHX group compared to the laser group and similar to the PDT group. ${ }^{18}$

In other study conducted on SLA implants, the use of Er:YAG laser irradiation resulted in statistically significantly superior biofilm removal compared to the 3 other treatments (titanium curettes, PDT and curettes with adjunctive PDT). The study also proved no statistically important differences in the reattachment of epithelial cells (EC), gingival fibroblasts and osteoblast-like cells to titanium SLA surfaces after each method of decontamination. ${ }^{19}$ To the best of our knowledge, there are very few 
studies in the field of decontamination which are similar to our work on different surface implants. One of the mentioned studies was carried on SLA, TPS and HA implants. After Er:YAG laser irradiation at pulse energies of $60 \mathrm{~mJ}$ and $120 \mathrm{~mJ}$ and at a frequency of $10 \mathrm{pps}$ led to bacterial reductions of $99.51 \%$ (SA), 98.39\% (HA) and 99.6\% (TPS) at a pulse energy of $60 \mathrm{~mJ}$, and $99.92 \%$ (SA), 99.85\% (HA) and $99.94 \%$ (TPS) at $120 \mathrm{~mJ} .^{20}$

\section{Conclusions}

The superiority of combined chemical-mechanical method of decontaminating the surface of an implant on SLA and machined-surface implants was proved. On the contrary, Er:YAG laser irradiation was reported as the best option for decontaminating the HA-coated implants.

\section{ORCID iDs}

Paweł Kubasiewicz-Ross (D) https://orcid.org/0000-0001-7305-7161 Małgorzata Fleischer (D) https://orcid.org/0000-0002-6610-3016 Artur Pitułaj (D) https://orcid.org/0000-0002-9025-2628 Jakub Hadzik (D) https://orcid.org/0000-0002-2353-3198 Izabela Nawrot-Hadzik (D) https://orcid.org/0000-0002-5797-7336 Olga Bortkiewicz (D) https://orcid.org/0000-0001-6122-5359 Marzena Dominiak (D) https://orcid.org/0000-0001-8943-0549 Kamil Jurczyszyn (D) https://orcid.org/0000-0002-0667-7261

\section{References}

1. Lindhe J, Meyle J. Peri-implant diseases: Consensus report of the sixth European workshop on periodontology. J Clin Periodontol. 2008;35 (8 Suppl):282-285

2. Mahato N, Wu X, Wang L. Management of peri-implantitis: A systematic review, 2010-2015. SpringerPlus. 2016;5:105.

3. Khammissa RA, Feller L, Meyerov R, Lemmer J. Peri-implant mucositis and peri-implantitis: Bacterial infection. SADJ. 2012;67(2):70-74.

4. Medina CMA, Villa-Correa YA. Gram-negative enteric rods associated to early implant failure and peri-implantitis: Case report and systematic literature review. Int J Odontostomat. 2015;9(2):329-336.

5. Leonhardt A, Dahlén G, Renvert S. Five-year clinical, microbiological, and radiological outcome following treatment of peri-implantitis in man. J Periodontol. 2003;74(10):1415-1422.
6. Saffarpour A, Nozari A, Fekrazad R, Saffarpour A, Heibati MN, Iranparvar K. Microstructural evaluation of contaminated implant surface treated by laser, photodynamic therapy, and chlorhexidine 2 percent. Int J Oral Maxillofac Implants. 2018;33(5):1019-1026.

7. Mengel R, Buns CE, Mengel C, Flores-de-Jacoby L. An in vitro study of the treatment of implant surfaces with different instruments. Int J Oral Maxillofac Implants. 1998;13(1):91-96.

8. Kuo HN, Mei HI, Liu TK, Liu TY, Lo LJ, Lin CL. In vitro laser treatment platform construction with dental implant thread surface on bacterial adhesion for peri-implantitis. Biomed Res Int. 2017;2017:4732302.

9. Suzuki JB. Salvaging implants with an Nd:YAG Laser: A novel approach to a growing problem. Compend Contin Educ Dent. 2015;36(10):756-761.

10. Arısan V, Karabuda ZC, Arıcı SV, Topçuoğlu N, Külekçi G. A randomized clinical trial of an adjunct diode laser application for the nonsurgical treatment of peri-implantitis. Photomed Laser Surg. 2015;33(11):547-554.

11. Kuroda K, Okido M. Hydroxyapatite coating of titanium implants using hydroprocessing and evaluation of their osteoconductivity. Bioinorg Chem Appl. 2012;2012:730693.

12. Shumaker ND, Metcalf BT, Toscano NT, Holtzclaw DJ. Periodontal and periimplant maintenance: A critical factor in long-term treatment success. Comp Contin Educ Dent. 2009;30(7):388-390,392,394 passim; quiz 407,418

13. Mellado-Valero A, Buitrago-Vera P, Solá-Ruiz MF, Ferrer-García JC. Decontamination of dental implant surface in peri-implantitis treatment: A literature review. Med Oral Patol Oral Cir Bucal. 2013;1;18(6): 869-876.

14. Subramani K, Wismeijer D. Decontamination of titanium implant surface and re-osseointegration to treat peri-implantitis: A literature review. Int J Oral Maxillofac Implants. 2012;27(5):1043-1054.

15. Meyle J. Mechanical, chemical and laser treatments of the implant surface in the presence of marginal bone loss around implants. Eur J Oral Implantol. 2012;5(Suppl):S71-81.

16. Blasi A, lorio-Siciliano V, Pacenza C, Pomingi F, Matarasso S, Rasperini G. Biofilm removal from implants supported restoration using different instruments: A 6-month comparative multicenter clinical study. Clin Oral Impl Res. 2016;27(2):e68-73.

17. Dennison DK, Huerzeler MB, Quinones C, Caffesse RG. Contaminated implant surfaces: An in vitro comparison of implant surface coating and treatment modalities for decontamination. J Periodontol. 1994; 65(10):942-948.

18. Marotti J, Tortamano P, Cai S, Ribeiro MS, Franco JE, de Campos TT. Decontamination of dental implant surfaces by means of photodynamic therapy. Lasers Med Sci. 2013;28(1):303-309.

19. Eick S, Meier I, Spoerle F, et al. In vitro-activity of Er:YAG laser in comparison with other treatment modalities on biofilm ablation from implant and tooth surfaces. PLoS One. 2017;26;12(1):e0171086.

20. Kreisler M, Kohnen W, Marinello $C$, et al. Bactericidal effect of the Er:YAG laser on dental implant surfaces: An in vitro study. J Periodontol. 2002;73(11):1292-1298. 